\title{
Hypopigmentation with muscular and subcutaneous atrophy as a complication of Intralesional Steroid injection in Ganglion
}

\author{
Sandip Kumar Rahul a, ${ }^{\mathrm{a}}$, Digamber Chaubey ${ }^{\mathrm{a}}$, Rupesh Keshri ${ }^{\text {a }}$, Pallavi Suman ${ }^{\text {a }}$ \\ ${ }^{a}$ Department of Pediatric surgery, Indira Gandhi Institute of Medical Sciences, Patna, India.
}

\section{Dear Sir,}

Although ganglion cysts are commonly encountered in surgical practice, recurrences have been reported with almost all modalities used in its management ${ }^{[1]}$. We report an unusual complication following intra-lesional steroid injection. Consent was taken from the father of the patient before presenting this case; permission was also taken from the Institutional Ethics Committee (Letter No. 106/IEC/2021/IGIMS, dated 12/05/2021). A ten-year-old-male presented with a ganglion cyst over the dorsum of the right hand which was treated with aspiration and intralesional Triamcinolone injection. Lesion disappeared when he came for follow-up after one month. He, however, developed hypopigmentation at the site of injection of steroid along with atrophy of the dorsal forearm muscles and subcutaneous tissue which became more obvious after five months of the procedure (Figure 1). There was no deficiency in the movements or sensation of the hand or forearm as per the patient but on examination; we found that extension at the wrist joint on the involved side was weaker. What could have caused this complication? Local spillage of steroid, over-dosage, the effect of the medication (more than anticipated) and nerve injury could all be the cause. Lymphatic channels have an important role in returning macromolecules from the interstitial fluid into the venous circulation. Triamcinolone, being a macromolecule takes the lymphatic pathway but it also finds its way out due to its lipophilic

\footnotetext{
* Corresponding author: Sandip Kumar Rahul

Mailing address: Department of Pediatric surgery, Indira Gandhi Institute of Medical Sciences, Patna, India.

E-mail: sandeep.rahul65@gmail.com

Received: 15 April 2021 / Accepted: 26 May 2021
}

property by the virtue of the acetonide group between carbon-15 and 16 in its structure ${ }^{[2,3]}$. So, Injected Triamcinolone takes the lymphatic pathway and may come out at places to cause hypopigmentation and localized atrophy as seen in our case. It has been found that the number of melanocytes is normal in such cases of steroid-induced hypopigmentation but it appears that their functioning is suboptimal ${ }^{[4]}$.

As far as muscle atrophy and myopathy following steroid usage is concerned, several investigators have reported the atrophy of Type $2 \mathrm{~b}$ fibers (fast-twitch) fibers on exposure to steroids without any inflammation or necrosis and that it is more with the use of fluorinated steroids like Triamcinolone as in our case ${ }^{[5,6]}$. Multiple mechanisms have been suggested to explain steroid-induced myopathy ${ }^{[7]}$. However, muscle proteolysis mainly through the ubiquitin-proteasome system is the most important mechanism ${ }^{[7]}$. Several investigators have observed muscular, subcutaneous and fat atrophy following local steroid injection ${ }^{[8,9]}$.

On the dorsum of the hand, the scapholunate ligament is the most important site for the occurrence of ganglions ${ }^{[10]}$. This was the site of the ganglion in our patient. Muscular atrophy which followed intra-lesional steroid injection predominantly involved the Extensor digitorum, Extensor pollicis longus and Extensor pollicis brevis. These affected the extensor movement against resistance at the wrist joint and of the thumb and other digits. However, the patient didn't complain of any difficulty in his daily work. For these findings, the patient was evaluated by the physiotherapy team who recommended passive range of motion movements, followed by active exercises and then resistance exercises involving the extensors of the wrist and the metacarpophalangeal and interphalangeal joints of the involved digits. The patient did well with this exercise regime. There are encouraging results of local electri- 


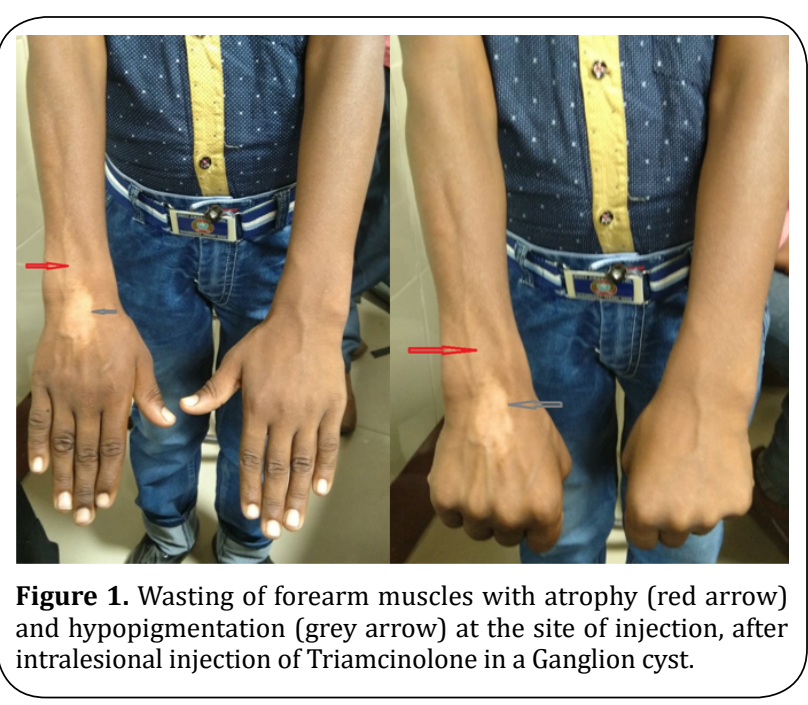

cal stimulation or focussed ultrasonic stimulation of atrophic muscles in stimulating the atrophic muscles to gain bulk and strength ${ }^{[11,12]}$. In our patient, local electrical stimulation was used initially for a month on alternate days and then exercise therapy was continued.

For subcutaneous fat atrophy, if it doesn't get corrected by itself after around a year of observation, the local fat injection has been tried successfully ${ }^{[13]}$. Shumaker et. al. discussed the positive role of local normal saline infiltration in four cases of persistent Cutaneous atrophy following steroid injection ${ }^{[9]}$. Our patient didn't need any such intervention for fat or cutaneous atrophy.

Ganjoo et. al. reported a linear streak of depigmentation following a single intralesional Triamcinolone injection in a ganglion over the anterior aspect of the wrist ${ }^{[14]}$. A similar pattern of hypopigmentation has been described by other investigators ${ }^{[2,4]}$. Venkatesan et. al. reported linear hypopigmentation and cutaneous atrophy following intra-articular steroids for De Quervain's tendonitis ${ }^{[4]}$. For hypopigmentation, our patient received $0.1 \%$ tacrolimus and 8 - methoxy Psoralen topically after consulting a dermatologist.

We have had recurrences with all modalities of treatment (surgery, simple aspiration, intralesional injection of steroids or sclerosants and closed rupture of these cysts) at our center and we choose the appropriate intervention after discussing with the relatives all these options including their complications. We have used aspiration with intralesional steroid injection using a single dart technique after appropriate counseling in a few of our patients who have not chosen an invasive procedure like surgery for management. In this case, Parents refused to get the involved site biopsied. This patient was then managed by the combined efforts of the dermatologists and Physiotherapists. After 20 months of intralesional injection, the wasted muscles regained their original shape with regular physiotherapy but some hypopigmentation has still persisted.

We concluded that intralesional steroid injection may result in significant hypopigmentation, atrophy of subcutaneous tissue and fat and muscular wasting at sites distant to the injection site and these complications along with high recurrence rate must be taken into consideration while planning management of a Ganglion cyst.

\section{DECLARATIONS}

\section{Authors' contributions}

All the authors were involved in the management of the patients; Sandip Kumar Rahul and Digamber Chaubey also did the framing of the manuscript; all the authors have read and approved the manuscript before submission.

\section{Conflict of interest}

All authors declared that there are no conflicts of interest.

\section{Ethics approval}

Consent was taken from both parents and the child before submitting the case and clinical pictures.

\section{REFERENCES}

1. Chatterjee, S., Basu, A., Gupta, S., \& Biswas, S. (2014). Comparative study of recurrence and complications using various sclerosants by single dart technique in treatment of ganglion cysts. Indian Journal of Surgery, 76(5), 350-353.

2. Prasad, K., Madke, B., Kar, S., Sinha, A. K., \& Yadav, N. (2015). Linear rays of depigmentation along lymphatics after intralesional corticosteroid therapy. Indian dermatology online journal, 6(6), 456-457.

3. Chembolli, L., Rai, R., \& Srinivas, C. R. (2008). Depigmentation along lymphatic channels following intralesional corticosteroid injection. Indian journal of dermatology, 53(4), 210.

4. Venkatesan, P., \& Fangman, W. L. (2009). Linear hypopigmentation and cutaneous atrophy following intra-articular steroid injections for de Quervain's tendonitis. Journal of drugs in dermatology: JDD, 8(5), 492-493.

5. Surmachevska, N., \& Tiwari, V. (2020). Corticosteroid Induced Myopathy. StatPearls [Internet].

6. Owczarek, J., Jasiñska, M., \& Orszulak-Michalak, D. (2005). 
Drug-induced myopathies. An overview of the possible mechanisms. Pharmacol Rep, 57(1), 23-34.

7. Schakman, O., Gilson, H., \& Thissen, J. P. (2008). Mechanisms of glucocorticoid-induced myopathy. The Journal of endocrinology, 197(1), 1-10.

8. Park, S. K., Choi, Y. S., \& Kim, H. J. (2013). Hypopigmentation and subcutaneous fat, muscle atrophy after local corticosteroid injection. Korean journal of anesthesiology, 65(6 Suppl), S59.

9. Shumaker, P. R., Rao, J., \& Goldman, M. P. (2005). Treatment of local, persistent cutaneous atrophy following corticosteroid injection with normal saline infiltration. Dermatologic surgery, 31(10), 1340-1343.

10. Angelides, A. C., \& Wallace, P. F. (1976). The dorsal ganglion of the wrist: its pathogenesis, gross and microscopic anatomy, and surgical treatment. The Journal of hand surgery, 1(3), 228-235.
11. Kus, G., \& Yeldan, I. (2019). Strengthening the quadriceps femoris muscle versus other knee training programs for the treatment of knee osteoarthritis. Rheumatology international, 39(2), 203-218.

12. Bueno, C. R. D. S., Pereira, M., Favaretto Junior, I. A., Bortoluci, C. H. F., Santos, T. C. P. D., Dias, D. V., ... \& Rosa Junior, G. M. (2017). Electrical stimulation attenuates morphological alterations and prevents atrophy of the denervated cranial tibial muscle. Einstein (Sao Paulo), 15(1), 71-76.

13. Imagawa, K., \& Ohkuma, S. (2010). A case of fat injection for treating subcutaneous atrophy caused by local administration of corticosteroid. Tokai J Exp Clin Med, 35(2), 66.

14. Shikhar, G., \& Vinita, G. (2018). Perilymphatic Linear Depigmentation in a Child following intralesional Steroid for Ganglion. Archives of Dermatology and Skin Care. 1(2), 1-2. 